\title{
Editorial: The Complex Systems Section of EPJ B
}

EPJ $B$ now receives more than 1000 submissions per year, and accounting for almost half of these, the 'Complex Systems' section has become popular to a degree where we are proud to offer you for the first time a regular issue of the journal containing original papers only from this area. They were not specially submitted for this issue, but instead were all recently accepted and selected from among our growing set of regular submissions from the fields of statistical, nonlinear and interdisciplinary physics.

This is thus not a typical topical issue, and all the papers presented here have individually followed the regular procedure of the standard peer-reviewing process - with at least two independent referee reports - sometimes with intense scientific arguments exchanged between authors, editors and referees prior to their final acceptance.

Yet this regular issue is quite a 'special' one, in different respects. The 'Complex Systems' section was launched in 2007. Yet after only two years with the extended scope of EPJ B, both the publishers and the editors feel that we have good reasons to summarize and promote the recent achievements resulting from this expansion. As mentioned before, around half of all submissions to EPJ B are now assigned to the 'Complex Systems' section with roughly equal share between the two subsections 'Statistical and Nonlinear Physics' and 'Interdisciplinary Physics'. With a current rejection rate of almost 60 percent, EPJ $B$ publishes some 220 papers per year in these areas. This follows a clearly increasing trend, notwithstanding the consensus to keep the rejection rate high, given the aim to establish $E P J B$ as the leading physics journal in the field.

In the context of this optimistic perspective, it is worth remembering the benefits of having the two sections 'Condensed Matter' and 'Complex Systems' next to each other in one journal. Beyond the historical arguments reflecting the evolution of the journal themes over time, the major reason is their common shared methodology. Most of the theoretical methods and tools used for the investigations of complex systems have their origin in statistical physics as it was developed originally to describe collective phenomena in many particle systems. Even if, over the last 20 years, the complex systems research community has established their own approaches to the wide range of collective phenomena found outside of condensed matter physics, there is still the possibility to transfer knowledge regarding analytical and numerical methods, and in this way to provide inspiration for research in both domains.

The current selection of regular submissions to the 'Complex Systems' section of EPJ B highlights some of the trends in this field. 'Complex Networks', a booming research area for several years now [cf. also the Topical Issue 'Applications of Networks' in Eur. Phys. J. B 38, 141-391 (2004)] was the dominant topic in the submissions over the last two years, which is also reflected by the number of papers in this issue. The research in this field is increasingly driven by data available from social and economic systems, in addition to extending theoretical concepts to characterize these networks.

Another major branch of submissions deals with issues of 'sociophysics', such as opinion dynamics or evolution of cooperation. Many of these papers deal with agent-based approaches - a generalization of many-particle systems. However, different from most physical systems, agents in social or economic systems are not identical but 'heterogeneous', their interactions lack symmetries and change over time, based on experience and external feedbacks. This may indicate some limitations for the 'simple' transfer of methods from statistical physics into 'living' systems, but also provides new challenges for the development of appropriate concepts.

Econophysics, now becoming an increasingly established field, as well as the physics of traffic, are still attractive areas of complex systems research, however with a declining number of submissions. This can be taken as a sign of consolidation in these areas - to make progress, now, it takes more than a few new computer simulations or the 
correlation analysis of stock market data. Investigations by physicists in these areas should be possibly rooted in analytical approaches, if not in fundamental theories established in these areas.

In addition to the elaborate topics of some papers dealing with interdisciplinary physics, a good fraction of the submissions also focus on more classical problems of Complex Systems, such as pattern formation, phase transitions, and non-linear time series analysis. As these topics are commonly represented in many physics journals, there is of course a larger competition among journals for the best papers, and EPJ B is proud to be now a well established and respected publishing platform also for such "core physics" papers.

This issue also includes three papers by physicists focusing on the statistics of sports such as soccer and baseball. Are these complex systems? Is this physics? We are not going to give a definite answer to these questions, but the three different perspectives taken on these issues are not just informative but also quite entertaining.

Last but not least, this selection of papers from the current submissions to EPJ B in the form of a 'special issue', is presented with the explicit aim and wish to contribute to the effort of a whole community to promote the exciting and burgeoning field of physics of complex systems. We hope to inspire and hook scientists and graduate students from related disciplines and to encourage them to start their own research in this direction. Of course, we cordially invite all of them to submit their best manuscripts in this area to EPJ $B$. We provide a fair, personalized and fast refereeing process, where the papers are handled by editors who are active and internationally recognized researchers in their respective fields.

I 'd like to wish you many interesting moments, inspiration, and just plain 'scientific entertainment' when reading through this 'special' regular issue of EPJ $B$.

Editor-in-Chief

Frank Schweitzer 